\title{
ACCESSIBILITY SPACES
}

GORDON T. WHYBURN ${ }^{1}$

1. Introduction. For any class $\mathcal{C}$ of sets in a topological space $Y$ a limit point $p$ of a set $M$ is accessible by $\mathcal{C}$-sets provided $p$ is a limit point of a $\mathfrak{C}$-set lying in $M+p$. For example, if $\mathfrak{e}$ is the class of simple arcs or continua, we have the classical type of accessibility of boundary points of a region. The property of a $T_{1}$-space $Y$ to have every limit point of a set $M$ accessible by closed sets in this sense was introduced by the author in 1956 [16] and called property $H$. It was shown that any quasi-compact (=quotient) mapping onto a range space $Y$ having this property is hereditarily quasi-compact (=hereditarily quotient $[12]=$ pseudo-open [2] = quasi-compact on every inverse set). In the present paper it is shown that this same property exactly characterizes those regular $T_{1}$-spaces as range spaces onto which all quasi-compact mappings are hereditarily quasi-compact. In other terms, we show that: $A$ regular $T_{1}$-space $Y$ has the property that each limit point of a set in $M$ is accessible by closed sets if and only if every quasi-compact mapping onto $Y$ is hereditarily quasi-compact.

A slight variation of the property yields a similar characterization for such range spaces without the regularity condition. We also discuss briefly some equivalences among the bewildering multitude of related concepts and terms involving spaces and mappings of this sort which have been introduced and studied in recent years. For example, property $K$ of Halfar [7], which is equivalent to the property of being an hereditary $k$-space, says that each limit point of a set $M$ is accessible by compact sets.

A $T_{1}$-space $Y$ will be called an accessibility space provided every limit point $p$ of a set $M$ in $Y$ is approximately accessible by closed sets, that is, there exists a closed set $C$ having $p$ as a limit point and such that all points of $C$ in some neighborhood of $p$ lie in $M+p$. In other words, $p$ is a limit point of $C$ but not of $C-M$. It is clear that for regular spaces this is exactly the same property $H$ above of having all limit points of sets accessible by closed sets and that, in any case, it is always implied by property $H$. Note: The term accessibility space as used here should not be confused with the notion of accessible space as used by Fréchet in the early development of abstract space topology.

Received by the editors May 31, 1969.

1 This paper is being published posthumously. Professor Whyburn died on September 8, 1969. 
2. THEOREM. In order that a $T_{1}$-space $Y$ be an accessibility space it is necessary and sufficient that every quasi-compact mapping onto $Y$ be hereditarily quasi-compact.

Suppose first that $Y$ is an accessibility space. We have to show that if $f: X \rightarrow Y$ is a quasi-compact mapping of a topological space $X$ into $Y$, then $f$ is quasi-compact on every inverse set of $f$. To this end let $A=f^{-1}(B), B \subset Y$, be an inverse set and suppose, contrary to our assertion, that some inverse set $N$ in $A$ which is closed relative to $A$ has image set $M$ which is not closed in $B$. Thus there exists a point $p \in B-M$ which is a limit point of $M$. By the accessibility condition there exists a closed set $C$ such that $p$ is a limit point of $C$ but not of $C-M$, so that the set $D=\operatorname{cl}(C-M)-p$ is closed and does not contain $p$. Then since $N$ is closed in $A$, neither of the closed sets $\bar{N}$ and $f^{-1}(D)$ meets $f^{-1}(p)$. Accordingly

$$
f^{-1}(C)-f^{-1}(p)=\bar{N} \cdot f^{-1}(C)+f^{-1}(D) .
$$

This set is therefore a closed inverse set with a nonclosed image set $C-p$, contrary to quasi-compactness of $f$.

To prove the converse, we suppose that the $T_{1}$-space $Y$ is not an accessibility space. Then there exists a set $M$ in $Y$ and a limit point $p$ of $M$ in $Y-M$ such that $p$ is not a limit point of any closed set all points of which in some neighborhood of $p$ lie in the set $M+p$. In other words, if $p$ is a limit point of a closed set $C$, then it is also a limit point of $C-M$. We proceed to set up a domain space $X$ and a mapping $f: X \rightarrow Y$ of $X$ onto $Y$ which is quasi-compact but fails to be quasi-compact on its kernel. ${ }^{2}$

The space $X$ is a subset of the product space $Y \times\{0,1\}$. The copies in $Y \times\{0\}$ and $Y \times\{1\}$ of any set $E$ in $Y$ will be denoted by $E_{0}$ and $E_{1}$ respectively. First we define $N=Y-M-p$, so that $Y=M+N+p$ $\approx Y \times\{0\}=Y_{0}$. Now $N_{1}=N \times\{1\}$ and $p_{1}=(p, 1)=\{p\} \times\{1\}$ and we define $X=N_{1}+p_{1}+M_{0}+N_{0}=(N+p) \times\{1\}+(Y-p) \times\{0\}$, where $X$ has the relative topology in the product space. Finally we define the map $f$ from $X$ to $Y$ by $f(x, t)=x$ for $(x, t) \in X$, Noting that $N^{1}+p^{1}$ and $(M+N)_{0}$ are disjoint closed sets in $X$, we observe at once:

(i) $f \mid(M+N)_{0}$ is a homeomorphism onto $M+N$;

(ii) $f \mid\left(N_{1}+p_{1}\right)$ is a homeomorphism onto $N+p$;

(iii) the kernel of $f$ is $M_{0}+p_{1}$.

Thus $f$ is a mapping of $X$ onto $Y$. Further, $f \mid\left(M_{0}+p_{1}\right)$ is clearly not

${ }^{2}$ The kernel of a mapping $f: X \rightarrow Y$ is the set of all $x \in X$ satisfying the relation $x$ $=f^{-1} f(x)$. 
quasi-compact because $M_{0}$ is closed relative to $M_{0}+p_{1}$ as a subset of $X$ but $M$ is not closed relative to $M+p$ as a subset of $Y$. (Simpler, note that $M_{0}+p_{1}$ in $X$ is not homeomorphic with $M+p$ in $Y$.)

Thus we have left only to show that $f$ itself is quasi-compact. To that end, take a closed inverse set $E$ in $X$. Then by observations (i) and (ii) it follows that $f(E)$ is closed except possibly for $p$. Hence if $p_{1} \in E, p \in f(E)$ and $f(E)$ is closed. Hence we assume $p_{1}$ not in $E$. Now $f\left(E \cdot N_{1}\right)$ is closed in $N+p$ as a subset of $Y$; and it does not contain $p$ because $p_{1}$ is not in $E$. Thus there is an open set $U$ in $Y$ about $p$ not meeting this set. This gives $f(E) \cdot U \subset M$. Hence if we define $C$ $=\operatorname{cl}(f(E)) \cdot \bar{U}$, then $C$ is closed and $p$ is not a limit point of $C-M$ because $C \cdot U \subset M+p$. Accordingly $p$ is not a limit point of $C$ and hence also not of $f(E)$. Thus $f(E)$ is closed, as was to be shown.

3. Consequences. The theorem and proof just given in $\$ 2$ yield the following corollaries.

(3.1). If $Y$ is any nonaccessibility $T_{1}$-space, there exists a subset $X$ of the product space $Y \times\{0,1\}$ such that the projection $f$ of $X$ into $Y$ is onto and quasi-compact but it not quasi-compact on its kernel.

It may be noted here that if $Y$ is regular or Hausdorff so also is $X$. Also $f$ is an at most two to one mapping which is a homeomorphism on each of two disjoint complementary closed sets in $X$.

(3.2). If every quasi-compact mapping onto a $T_{1}$-space $Y$ is quasicompact on its kernel, then every such mapping is hereditarily quasicompact.

Since for 1-1 mappings, quasi-compactness is the same as continuity for the inverse function, we have

(3.3). In order that a $T_{1}$-space $Y$ be an accessibility space it is necessary and sufficient that every quasi-compact mapping into $Y$ be a homeomorphism on its kernel.

4. Space properties. Some equivalences. For simplicity in this discussion we assume all spaces are Hausdorff spaces. We consider the following properties:

(a) $k$ (Hurewicz): a set is closed if and only if it meets every compact set $C$ in a set which is closed in $C$.

(b) Fréchet [2]: a point $p$ is in the closure of a set $C$ if and only if some sequence in $C$ converges to $p$. (Also see [9].)

(c) $k^{\prime}[3]$ : each limit point of a set $M$ is a limit point of the intersection of $M$ with some compact set. (See also [6], [18].) 
(d) $K[7]$, or $k_{1}$ [6]: each limit point of a set is accessible by compact sets.

(e) sequential [5]: a set is open if and only if each sequence converging to a point of the set is eventually in the set.

(f) hereditarily $k$ : every subset is a $k$-space.

(g) hereditarily $k^{\prime}$ : every subset is a $k^{\prime}$-space.

(h) hereditarily sequential: every subset is a sequential space.

(4.1). Theorem. For Hausdorff spaces the properties (d), (f), (g), (b) and (h), i.e., $K$, hereditarily $k$, hereditarily $k^{\prime}$, Fréchet and hereditarily sequential, are all equivalent. Further, each of them implies that the space is an accessibility space.

That (d), (f) and (g) are mutually equivalent is readily proven. Likewise for the equivalence of (b) and (h) (see [5]). However the remaining bridge between these two groups of properties presents some difficulty. This has been overcome, independently, by Arhangel'skil [4] who proved hereditarily $k$ equivalent to the Fréchet property, and by Mary Ellen Rudin (her proof is given in [11]) who proved $K$ equivalent to Fréchet.

5. Mapping properties. Again our discussion is limited to onto mappings with Hausdorff spaces as domain and range. Some years ago A. H. Stone [14] pointed out equivalences between a number of mapping properties and identical properties with different names then in use. Since that time the terminology has grown even more diverse.

In general it is fairly well known that the properties: Strong continuity (Alexandroff-Hopf [1]), quasi-compact (Whyburn [17]), quotient (Michael [12], Hu [8], Bourbaki, and others), factor (Arhangel'skiı̆ [2]), identification ( $\mathrm{Hu}[8]$ and others), and decomposition, are all identical.

Less known, however, is the fact that the properties: $P_{1}$ (McDougle [10]), pseudo-open (Arhangel'skiǐ [2]), preclosed (T'ong [15]), hereditarily quotient (Michael [12]), and hereditarily quasi-compact, are all equivalent - the first three having identical definitions as do also the last two. That the last four of these are equivalent seems generally known (see [2] for example), but that a study of this property appeared in McDougle's 1958 paper [10] seems to have escaped notice by several recent writers.

It may also be pointed out that the properties: $P_{2}$ (McDougle [10]), biquotient (Michael [12]), compact trace property (Whyburn [19]), and compact covering property (Michael [13]), are very close 
to each other. Although they are not entirely equivalent in the most general Hausdorff space situation they become so when the domain or range or the mapping is somewhat restricted. Conceptually they are much the same.

\section{REFERENCES}

1. P. Alexandroff and H. Hopf, Topologie, Springer-Verlag, Berlin, 1935, reprint, Chelsea, New York, 1965. MR 32 \#3023.

2. A. V. Arhangel'skil, Some types of factor mappings and the relations between classes of topological spaces, Dokl. Akad. Nauk SSSR 153 (1963), 743-746=Soviet Math. Dokl. 4 (1963), 1726-1729. MR 28 \#1587.

3. - Bicompact sets and the topology of spaces, Dokl. Akad. Nauk SSSR 150 (1963), 9-12 =Soviet Math. Dokl. 4 (1963), 561-564. MR 27 \#720; MR 28 p. 1246.

4. ——, A characterization of very $k$-spaces, Czechoslovak Math. J. 18 (93) (1968), 392-395. MR 37 \#4768.

5. S. P. Franklin, Spaces in which sequences suffice, Fund. Math. 57 (1965), 107116. Also, Spaces in which sequences suffice. II, Fund. Math. 61 (1967), 51-56. MR 31 \#5184; MR 36 \#5882.

6. R. V. Fuller, Relations among continuous and various non-continuous functions, Pacific J. Math. 25 (1968), 495-509. MR 37 \#3536.

7. Edwin Halfar, Conditions implying continuity of functions, Proc. Amer. Math. Soc. 11 (1960), 688-691. MR 22 \#8474.

8. S. T. Hu, Introduction to general topology, Holden-Day, San Francisco, Calif., 1966. MR $33 \# 4876$.

9. W. E. Malbon, Invariants for quasi-compact mappings, Dissertation, University of Virginia, Charlottesville, Va., 1955.

10. P. McDougle, $A$ theorem on quasi-compact mappings, Proc. Amer. Math. Soc. 9 (1958), 474-477. MR 20 \#1971.

11. Evelyn R. McMillan, On continuity conditions for functions, Dissertation, University of Virginia, Charlottesville, Va., 1968.

12. E. Michael, Bi-quotient maps and cartesian products of quotient maps, Ann. Inst. Fourier (Grenoble) 18 (1968), 287-302.

13. - Local compactness and cartesian products of quotient maps and $k$-spaces, Ann. Inst. Fourier (Grenoble) 18 (1968), 281-286.

14. A. H. Stone, Metrizability of decomposition spaces, Proc. Amer. Math. Soc. 7 (1956), 690-700. MR 19, 299.

15. Din'N'e T'ong, Preclosed mappings and Taimanov's theorem, Dokl. Akad. Nauk SSSR 152 (1963), 525-528=Soviet Math. Dokl. 4 (1963), 1335-1338. MR 28 \#5427.

16. Gordon T. Whyburn, Mappings on inverse sets, Duke Math. J. 23 (1956), 237-240. MR 20 \#4822. 194.

17. - Open and closed mappings, Duke Math. J. 17 (1950), 69-74. MR 11,

18. - Directed families of sets and closedness of functions, Proc. Nat. Acad. Sci. U.S.A. 54 (1965), 688-692. MR 32 \#435.

19. —-, Compactness of certain mappings, Amer. J. Math. 81 (1959), 306-314. MR $22 \# 1881$.

UNIVERSITY OF VIRGINIA 